\title{
Tuberculosis gastrointestinal como causa de hemorragia digestiva masiva en un paciente con infección por VIH
}

\author{
Omayra Chincha, Jaime Cáceres y Carlos Seas
}

\section{Gastrointestinal tuberculosis as a cause of massive gastrointestinal bleeding in a patient with HIV infection}

Gastrointestinal tuberculosis is a rare and fatal complication in patients with HIV infection. It occurs in 3\%-16\% of extra pulmonary forms. The main location in $90 \%$ of cases is the ileocecal valve and colon. The most unusual presentation of gastrointestinal tuberculosis is massive bleeding with the presence of one or multiple ulcers in the colon. The diagnosis can be confirmed by biopsy and by culture of intestinal tissue. Surgical resection is the treatment of choice. We report a case of a HIV-infected patient, who did not receive antiretrovirals, and who developed disseminated tuberculosis with intestinal perforation, presenting a fatal massive intestinal bleeding.

Key words: Mycobacterium tuberculosis; gastrointestinal tuberculosis; AIDS/HIV; gastrointestinal bleeding.

Palabras claves: Mycobacterium tuberculosis; tuberculosis gastrointestinal; VIH/SIDA; hemorragia digestiva masiva.

\section{Introducción}

L a tuberculosis (TBC) asociada a la infección por el virus de inmunodeficiencia humana (VIH) es uno de los mayores problemas de salud pública en el mundo ${ }^{1}$. La Organización Mundial de la Salud estima que $13 \%$ de los 8,6 millones de casos nuevos de TBC en el año 2012 fueron co-infecciones con VIH, causando 1,3 millones de muertes relacionados a $\mathrm{TBC}^{2}$. Los pacientes con ambas infecciones desarrollan 30 veces más TBC activa, predominando las formas extra-pulmonares. $\mathrm{La}$ TBC gastrointestinal es la sexta forma de presentación extra-pulmonar después de la linfática, genitourinaria, ósea, miliar y meníngea ${ }^{3}$. La TBC gastrointestinal se puede presentar como una enfermedad aguda o crónica. Su localización más frecuente es la región ileocecal y se presenta por lo general como lesiones ulceradas múltiples. La hemorragia digestiva es una presentación muy infrecuente ${ }^{4}$.

Se comunica el caso clínico de un paciente con infección por VIH de reciente diagnóstico, sin tratamiento anti-retroviral con una TBC gastrointestinal y pulmonar, que desarrolló una hemorragia digestiva masiva de curso fatal.

\section{Caso clínico}

Varón de 32 años, sin antecedentes mórbidos, que consultó por una pérdida de peso de $10 \mathrm{~kg}$ en un mes, asociado a un dolor abdominal tipo ardor que aumentaba con los alimentos y rectorragia. Se realizó una endoscopia digestiva alta donde se observó en la segunda porción del duodeno una úlcera de $8 \mathrm{~mm}$ con bordes definidos, con un lecho de fibrina. Hacia distal se encontró otra lesión exofítica con lecho ulceroso de $15 \mathrm{~mm}$ (Figura 1A). En la colonoscopia se evidenció en la válvula ileocecal una lesión ulcerada de $15 \mathrm{~mm}$ y varias úlceras con lecho de fibrina delgada, bordes elevados definidos en el ciego, colon descendente distal y sigmoides (Figura 1B). Se tomaron biopsia y cultivos de todas las lesiones. Entre los exámenes solicitados, destacaba ua hemoglobina de $6,9 \mathrm{~g} / \mathrm{L}$, hematocrito $20 \%$, leucocitos $6.530 / \mathrm{mm}^{3}, 88 \%$ neutrófilos segmentados, VHS $60 \mathrm{~mm} / \mathrm{h}$, PCR normal. La serología por técnica de ELISA para VIH resultó reactiva. El recuento de linfocitos T CD4 fue de 30 céls/ $\mathrm{mm}^{3}$ y la carga viral fue 859.841 copias $/ \mathrm{ml}$. El paciente no acudió a control por consulta externa de infectología (sin completar estudios para la infección por VIH) y gastroenterología. Posteriormente, 20 días después de la endoscopia, el paciente acudió a la Unidad de Emergencias con una historia de una semana de melena asociada a vómitos alimentarios, al los que finalmente se agregó dolor abdominal tipo cólico y hematoquecia.

El paciente ingresó con hipotensión arterial, con una presión: 80/40 mmHg, taquicárdico (144 por min), frecuencia respiratoria: 26 por min y temperatura de $38,6^{\circ} \mathrm{C}$. Con el diagnóstico de shock hipovolémico, se le administró hidratación enérgica con fluidos intravenosos y fármacos vasopresores. Al examen físico se evidenció
Universidad Peruana

Cayetano Heredia. Hospital Cayetano Heredia. Perú. Programa de Formación de Especialidad de Enfermedades Infecciosas y Tropicales (OCh). Anatomía Patológica (JC) Enfermedades Infecciosas y Tropicales (CS).

No existen conflictos de interés Fuente de Financiación: Ninguna.

Recibido: 24 de abril de 2017 Aceptado: 30 de mayo de 2017

Correspondencia a:

Omayra Chincha L.

omayra.chincha.l@upch.pe 
Figura 1. A: Endoscopia digestiva alta que muestra úlcera con lecho ulceroso con fibrina y bordes definidos, en segunda porción del duodeno. B: Colonoscopia con lesión ulcerada cecal de $15 \mathrm{~mm}$.
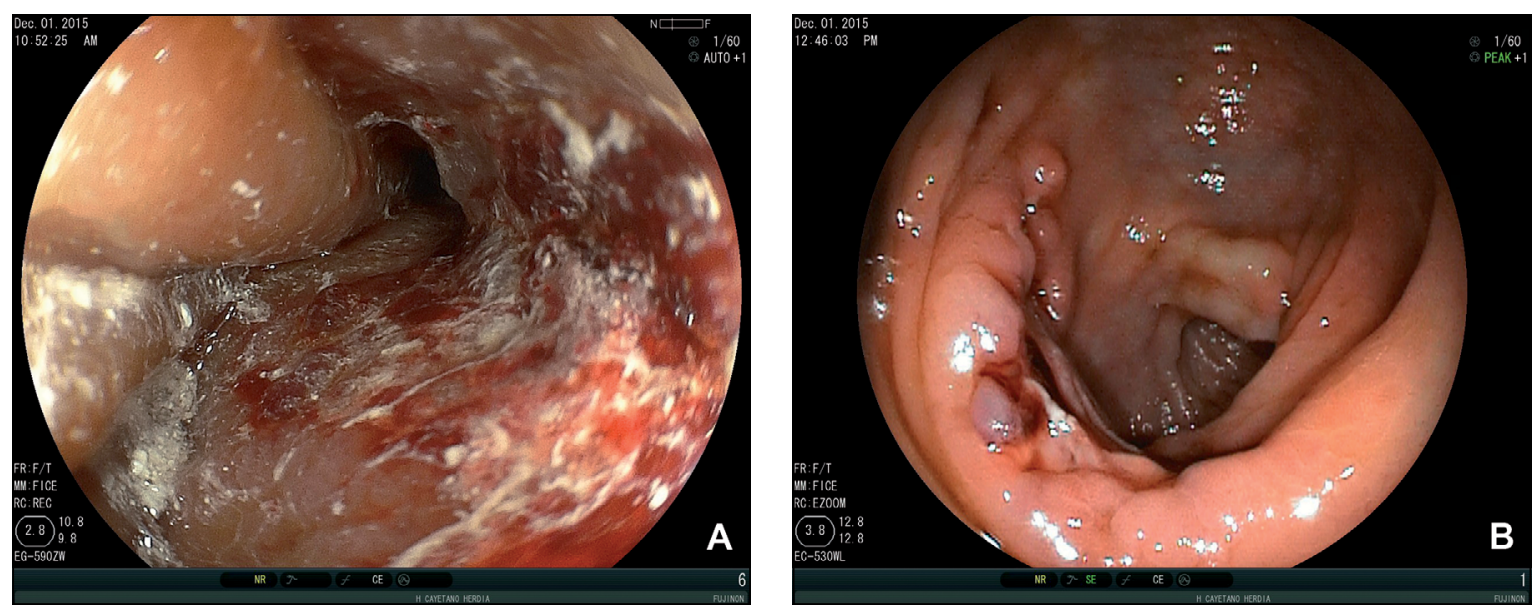

emergencia observándose una lesión ulcerada (Forrest Ib) en la segunda porción del duodeno con sangrado activo que se controló con adrenalina. El estudio histopatológico se informó como mucosa duodenal ulcerada con un proceso inflamatorio crónico tipo granulomatoso supurado que comprometía la mucosa y submucosa con zonas de necrosis. La coloración de PAS fue negativa y la tinción de Ziehl-Neelsen positiva. La mucosa colónica ulcerada mostró también un proceso inflamatorio crónico intenso de tipo granulomatoso, supurado, que comprometía la mucosa y submucosa, con coloración PAS negativa (Figura 3A) y Ziehl-Neelsen positiva (Figura 3B).

Con la histopatología de tejido intestinal y una TC compatible con una TBC miliar pulmonar, se inició tratamiento antituberculoso con isoniazida $5 \mathrm{mg} / \mathrm{kg}$, rifampicina $15 \mathrm{mg} / \mathrm{kg}$, etambutol $20 \mathrm{mg} / \mathrm{kg}$ y pirazinamida $25 \mathrm{mg} / \mathrm{kg}$. Los cultivos de tejido colónico y duodenal en el medio de Lowenstein fueron positivos para Mycobacterium tuberculosis susceptible a fármacos antituberculosos de primera línea. No se realizaron pruebas de sensibilidad rápidas, ni cultivos en medios líquidos. El paciente tuvo una evolución estacionaria; sin embargo, a las $48 \mathrm{~h}$ presentó un shock hipovolémico secundario a repetidos episodios de melena y hematemesis masiva refractarios al aporte de volumen intravenoso. Dada la condición crítica del paciente no fue posible una intervención quirúrgica de salvataje, falleciendo al décimo día de hospitalización.

\section{Discusión}

Este caso muestra una causa inusual de hemorragia digestiva alta y baja en un paciente con infección por VIH sin tratamiento anti-retroviral.

Las causas más frecuentes de hemorragia digestiva alta en un paciente con infección por VIH con recuento 

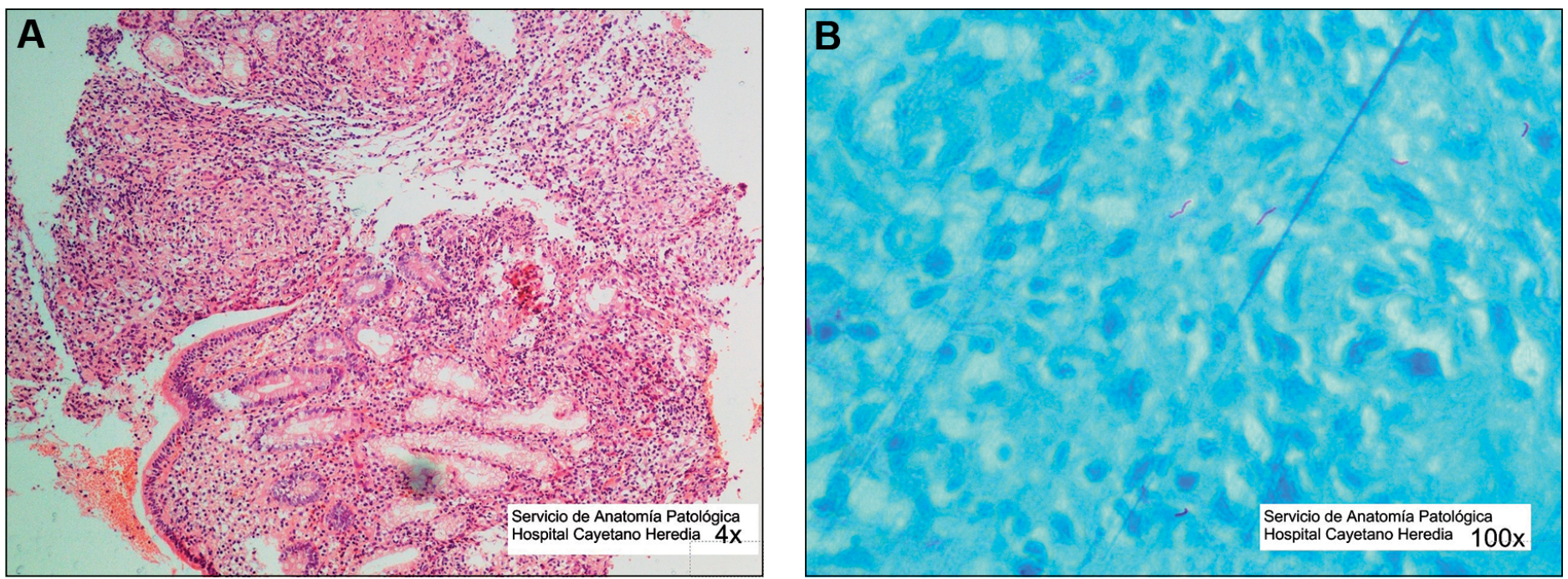

Figura 3. A: Mucosa duodenal ulcerada con proceso inflamatorio crónico tipo granulomatoso, supurado, que compromete la mucosa y submucosa con zonas de necrosis; B: Coloración Ziehl Neelsen positiva de la mucosa duodenal.

de linfocitos T CD4 $<200$ céls $/ \mathrm{mm}^{3}$ son el sarcoma de Kaposi, úlceras esofágicas virales (citomegalovirus, herpes simplex) y micóticas (candidiasis) y linfoma no Hodgkin $^{5}$, mientras que en el diagnóstico diferencial de sangrado digestivo bajo incluye: linfoma no Hodgkin, carcinoma, enfermedad diverticular e infecciones entéricas por Yersinia enterocolitica, Histoplasma capsulatum y citomegalovirus ${ }^{6}$. En el Perú y en otros países endémicos debe considerarse la TBC gastrointestinal dentro del diagnóstico diferencial para una hemorragia digestiva baja?

La TBC gastrointestinal se desarrolla por la deglución del esputo infectado o por diseminación de una enfermedad tuberculosa pulmonar. Así mismo se describe que en más de $25 \%$ de los pacientes con enfermedad cavitaria pulmonar se puede documentar una TBC gastrointestinal, pero sólo $20 \%$ de estos pacientes podría tener una infección pulmonar activa ${ }^{6}$. El proceso patológico inicial en la afectación gastrointestinal, después de la ingestión de esputo infectado, se inicia con la formación de tubérculos epitelioides localizados en el tejido linfático de la submucosa. Después de dos a cuatro semanas, la necrosis con caseificación ocasiona un desprendimiento de la mucosa suprayacente, lo que origina una úlcera en la mucosa ${ }^{8}$. La localización más frecuente es la región ileocecal, en $90 \%$ de los $\operatorname{casos}^{10}$, seguido de colon y yeyuno ${ }^{8}$.

La presentación clínica de la enfermedad se manifiesta con dolor abdominal asociado a otros síntomas constitucionales como fiebre, diarrea, pérdida de peso, anorexia y malestar general de varios meses de evolución ${ }^{3}$, como en el caso de nuestro paciente. El sistema inmune del hospedero influencia los patrones de la lesión: la forma ulcerativa se asocia a una evolución aguda en aquellos con la respuesta inmune disminuida, mientras que la forma hipertrófica ocurre en personas inmunocompetentes ${ }^{4}$. Las perforaciones intestinales pueden ser solitarias o múltiples y se producen entre 1 y $15 \%$ de los casos en el intestino delgado, generalmente en el íleon distal. Por el contrario, la perforación de colon de etiología tuberculosa es infrecuente ${ }^{8}$. Kawazoe y cols., comunicaron el caso de un paciente con infección por VIH con una una úlcera intestinal gigante y profunda asociada a una TBC miliar9 . La hemorragia digestiva masiva, definida como aquella que a pesar del aporte de líquidos y hemoderivados no posibilita estabilizar la hemodinamia, es de presentación infrecuente, constituyendo una verdadera emergencia, como en este paciente ${ }^{10}$.

Además del tratamiento específico antituberculoso, la resección quirúrgica debe realizarse en una lesión sangrante, dada la alta mortalidad asociada. Aunque la cirugía temprana mejora la sobrevida del paciente ${ }^{6-11}$, la decisión de intervenir dependerá del estado general del paciente, de la condición del tracto digestivo y el número de perforaciones. La perforación intestinal, aun con cirugía, puede ser fatal ${ }^{4}$. Nagashahi y cols., reportaron un paciente con infección por VIH de reciente diagnóstico con evidencia de sangrado intestinal bajo masivo, en el cual se realizó una cirugía de emergencia que logró controlar el sangrado. El paciente sobrevivió, a pesar de la mucosa debilitada del íleon y de las múltiples lesiones ${ }^{6}$. En nuestro paciente se tuvo que diferir la cirugía de emergencia dado el gran deterioro clínico; probablemente una cirugía temprana cuando se hizo el diagnóstico de úlceras intestinales hubiera mejorado el pronóstico.

El procedimiento diagnóstico de elección es usualmen- 
te la colonoscopia y la endoscopia digestiva alta con toma de muestras mediante biopsia. El diagnóstico definitivo de TBC gastrointestinal se establece por la tinción de Ziehl-Neelsen, cultivos convencionales en medio de Lowenstein Jensen y las características histopatológicas del tejido intestinal ${ }^{3-7}$.

\section{Resumen}

La tuberculosis (TBC) gastrointestinal es una complicación infrecuente y fatal en pacientes con infección por
VIH. Se presenta en 3-16\% de las formas extrapulmonares y su principal localización es la válvula ileocecal y colon. La hemorragia digestiva baja con presencia de una o múltiples úlceras en colon es una presentación muy inusual de TBC intestinal. El diagnóstico se confirma por biopsia y cultivo del tejido intestinal. La cirugía en caso de perforación intestinal sigue siendo el tratamiento de elección. Se presenta el caso de un paciente con infección por VIH, sin tratamiento anti-retroviral, quien desarrolló una TBC diseminada con perforación intestinal y hemorragia digestiva masiva de curso fatal.

\section{Referencias bibliográficas}

1.- Montales M T, Beebe A, Chaudhury A, Patil N Mycobacterium tuberculosis infection in a HIVpositive patient. Respir Med Case Rep 2015; 16: $160-2$.

2.- Lee S S, Lin H H, Tsai H C, Su I J, Yang C H, Sun H Y, et al. A clinical algorithm to identify HIV patients at high risk for incident active tuberculosis: a prospective 5 -year cohort study. PloS One 2015; 10: e0135801.

3.- Gómez-Zuleta M A, Viveros-Carreño D, Cañón D P. Tuberculosis intestinal: reporte de caso y revisión de la literatura. Infectio 2012; 16: 178-82.

4.- Hossein A, Sharifi M, Ahsan M, Afsharmoghaddam N. Multiple intestinal perforations as a primary manifestation of abdominal tuberculosis in a HIV-infected patient. JSCR 2010; 10: 7.

5.- Del Arco, De la Torre J, Merida L, Prada L, Pereda T, Rivera R, et al. Hemorragia digestiva alta en varón homosexual con infección por el virus de la inmunodeficiencia humana. Rev Clin Esp 2009; 209: 196-7.

6.- Nagahashi M, Aoyagi T, Yamada A, Rashid O M, Adams B J, Takabe K. Intestinal coinfection of tuberculosis and CMV can cause massive lower GI bleeding in a patient with HIV. J Surg Sci 2013; 1: 12-5.

7.- Piscoya A, Cedrón H, Huerta-Mercado J, Pinto J L, Ferrufino J C, Bussalleu A. GI tuberculosis and histoplasmosis in HIV (+) patient presenting with lower GI bleeding. Am J Gastroenterol 2005; 100: 1896-7.

8.- Tapias-Vargas L F, Santamaria C, Tapias-Vargas
L, Tapias L. Perforación de íleon terminal y ciego causada por tuberculosis intestinal en un paciente positivo para VIH. Rev Colomb Cir 2010; 25: 332-40.

9.- Kawazoe A, Nagata N. Intestinal tuberculosis in an HIV-infected patient with advanced immunosuppression. Clin Gastroenterol Hepatol 2012; 10: 24.

10.- Efsen A M, Schultze A, Post F A, Panteleev A, Furrer H, Miller R F, et al. Major challenges in clinical management of TB/HIV coinfected patients in Eastern Europe compared with Western Europe and Latin America. PloS One 2015; 10: e0145380.

11.- Villalon R, Rioseco M, Rubel S, Loyola C, Masia G. Úlcera tuberculosa intestinal como causa de hemorragia digestiva baja masiva exanguinante. Rev Chil Cir 2010; 62: 631-4. 